\section{Esophageal small-cell cancer mimicking stromal tumor}

A 59-year-old woman presented with progressive dysphagia. At esophagogastroduodenoscopy, a mass $4 \mathrm{~cm}$ long and $2 \mathrm{~cm}$ wide was observed in the cervical esophagus. It was a smooth-surfaced lesion, with blood vessels branching on top of it (Figure 1). An initial biopsy showed normal results, and the endoscopist suspected a stromal tumor. The patient was referred for an endoscopic ultrasound (EUS) examination, which was carried out with a miniprobe (Olympus UM-2R, $12 \mathrm{MHz}$ ). EUS revealed a homogeneous hypoechoic tumor arising from the mucosa and infiltrating the submucosa (Figure 2,3 ). This feature is atypical for a stromal tumor, which usually appears as a mass lesion with confined borders that arises from the submucosa or muscularis layers. EUS identified the mucosal part of the tumor, and repeated pinhole biopsies were taken.

The second histological assessment showed that the lesion was a small-cell cancer (Figure 4). Positron-emission tomography (PET) showed that there were no other primary or secondary tumor sites. The patient was diagnosed as having limited primary small-cell cancer in the esophagus. She received chemoradiotherapy treatment over a 3-month period. Follow-up PET and CT examinations 7 months later showed that the primary tumor had completely resolved, with the maximum standardized uptake value (SUV) having decreased from 9.4 to 3.1. However, the treatment was complicated by an esophageal stricture, which required repeated endoscopic dilation procedures. Repeated biopsies did not reveal any malignant cells.

Primary esophageal small-cell cancer is an uncommon condition, representing $1.0-2.8 \%$ of esophageal cancers $[1,2]$. Patients usually present late with anorexia, weight loss, and dysphagia due to tumor obstruction. To the best of our knowledge, there have been no previous reports in the literature on the EUS features of esophageal small-cell cancer. In this patient, the

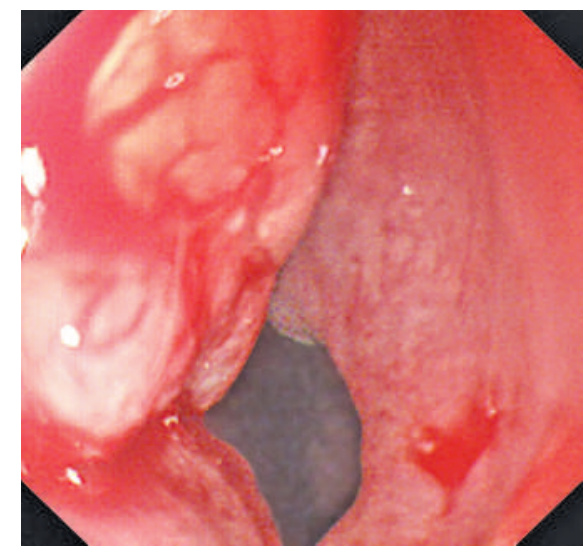

Figure 1 Esophagogastroduodenoscopy showed narrowing of the esophageal lumen due to a smooth-surfaced mass. There were dilated blood vessels on top of the mass.

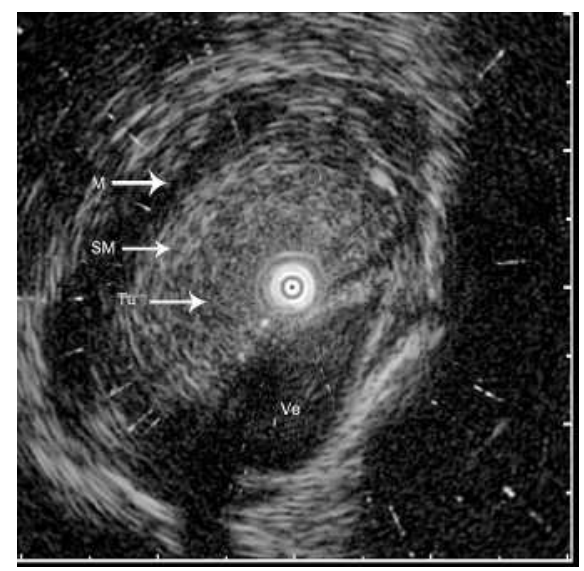

Figure 2 The endoscopic ultrasound miniprobe examination showed that the hypoechoic tumor (Tu) was infiltrating the hyperechoic submucosa (SM), without forming a defined margin. The muscle layer (M) was normal. A vessel (Ve) was noted outside the opposite wall.

lesion mimicked a stromal tumor. EUS raised the suspicion of malignant disease, which was subsequently confirmed by the guided biopsy.

Endoscopy_UCTN_Code_CCL_1AB_2AC_3AB

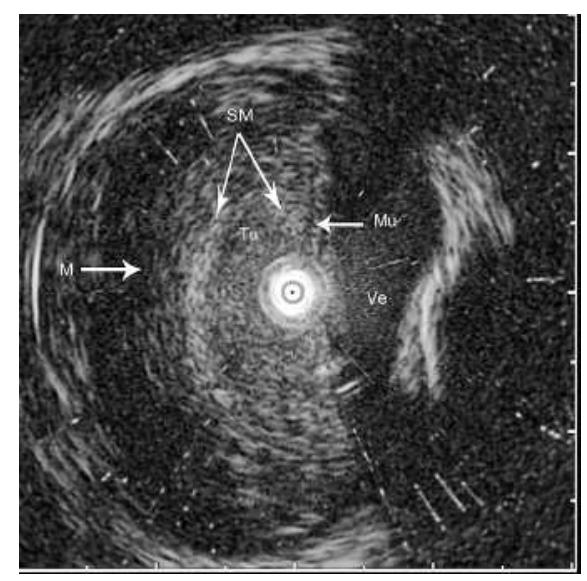

Figure 3 The mucosa (Mu) was intact over most of the tumor (Tu) site, as the tumor was mainly infiltrating the submucosa (SM), without breaking the mucosa. The muscle layer (M) was apparently thickened due to oblique scanning. A vessel (Ve) was noted outside the opposite wall.

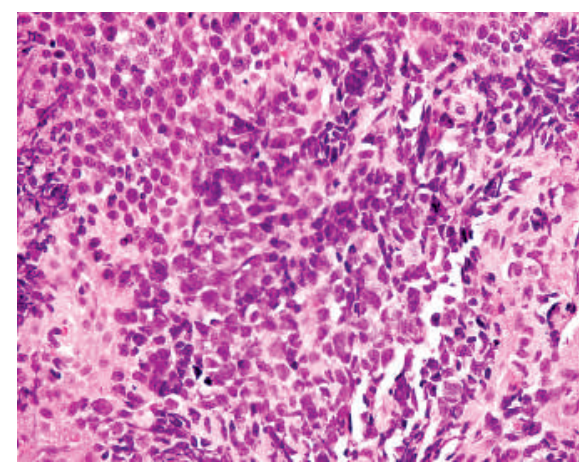

Figure 4 The histological analysis showed esophageal squamous epithelium in the upper left corner, with infiltrative sheets of small to medium-sized hyperchromatic malignant cells with stippled chromatin in the rest of the image (hematoxylin-eosin, original magnification $\times 40)$. 


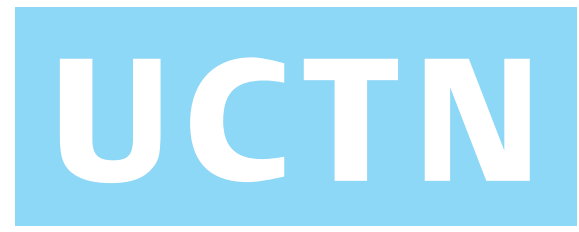

Y. T. Lee, P. W. Y. Chiu, P. C. L. Choi, J. J. Y. Sung

Institute of Digestive Diseases, Prince of Wales Hospital, Chinese University of Hong Kong, Shatin, N.T., Hong Kong SAR, China.
References

${ }^{1}$ Brenner B, Tang LH, Klimstra DS et al. Smallcell carcinomas of the gastrointestinal tract: a review. J Clin Oncol 2004; 22: 2730-2739

2 Wu Z, Ma JY, Yang JJ et al. Primary small cell carcinoma of esophagus: report of 9 cases and review of literature. World J Gastroenterol 2004; 10: 3680-3682
Corresponding author

Y. T. Lee, M.D.

Institute of Digestive Diseases

Prince of Wales Hospital

Chinese University of Hong Kong Shatin, N.T.

Hong Kong SAR

China

Fax: $\quad$ +852-2637-5396

E-mail: leeytong@cuhk.edu.hk 